circulating liquid. Details of the experiments will be communicated soon in the Zeitschrift für Physik.

The production of the magnetic fields can be easily understood, regarding the electrons as having a free mean path within the liquid mercury. The electrons will appear at any point $A$ with a velocity they have gained from the last impact at a different point $B$. Whenever a circulating flow of a liquid has a velocity gradient which is not linear, as is often the case in pipes, etc., the diffusion of the electrons causes an electric current in the direction of the flow and accordingly a magnetic field. For a rotational motion, the electric field producing the current observed cannot be compensated by the Coulomb forces of any space charges. Institut für Experimentalphysik,
Universität, Kiel.

$$
\begin{aligned}
& \text { F. BuRHoRN } \\
& \text { H. GRIEM } \\
& \text { W. LOCHTE-HoLtGREVEN }
\end{aligned}
$$
May 29.

\section{Magnetic Fields measured within Flames brought mechanically into Rotational Motion}

The flame of an oxygen burner was brought into spinning motion by forcing the gas tangentially into a cylindrical tube. Tho gas inlet is interrupted at frequent intervals in order to produce separate whirls of burning gas, which move along inside the tube one after the other. An induction coil surrounding the tube shows the existence of toroidal magnetic fields moving along together with (that is, surrounding) the plasma whirls. From the magnetic field we conclude that there exist electric currents within the plasma whirls accompanying the motion of the plasma.

Very careful experiments extending over several years show clearly that these currents can be attributed to the velocity difference of ions and electrons within each whirl. The electrons are retarded relatively to the ions because of their enhanced diffusion velocity into and back from cooler portions of the gas.

Quantitative measurements have been made in these experiments. From a determination of the temperature by several independent spectroscopic methods, the degree of ionization can be evaluated. An independent determination of the latter is obtained using the conductivity of the gas, which can also be measured and was found to reach values of the order of $10^{-11}$ e.s.u. if metallic potassium was introduced into the flame.

The induction coils were calibrated by means of a simple loop of wire, connected to an oscillatory device. For calibration the wire was put into the tube in place of the gas whirls. The measured toroidal magnetic field-strength created from the plasma whirls was found to be of the order of magnitude of $10^{-4}$ gauss.

The influence of magnetic fluctuations other than those created inside the tube was eliminated by using two identical coils connected in such a way that magnetic influence upon one coil only is to be registered. Compensation of the earth's magnetic field and application of an external field three times that of the earth causes only the zero level of our registration to shift up and down without altering the magnitude of the effects measured. Many other tests of the reality of the magnetic fields measured have been carried out.

Reduction of the speed of rotation causes the effect to decrease, becoming zero with no rotation at all. Decrease of the conductivity with less potassium added causes the effect to decrease likewise. Details of the experiments will be communicated in the Zeitschrift für Naturforschung.

The experiments described here and in the preceding communication deal with two different ways of creating magnetic fields within a 'plasma' by mechanical forces only. The first one deals with a plasma of practically zero compressibility, the latter with a compressible gas. In the latter, the experiments support the theory developed by Biermann and Schlüter ${ }^{1}$ et al. and are obviously analogous to astrophysical phenomena.

P. SCHILLING

W. LOCHTE-HoLTGREveN

Institut für Experimentalphysik, Universität, Kiel. May 29.

'Biermann, L., Z. Natf., 5a, 65 (1950). Biermann, L., and Schlüter, A., Phys. Rev., 82, $863(1951)$.

\section{Recovery of Isotopic Boron and Lithium from Nuclear Research Emulsions}

NuClear research emulsions are sometimes loaded with the stable isotopes of boron or lithium for work on nuclear disintegrations or on neutron counting by the photographic plate technique. Since the usual additions correspond to as much as $16 \mathrm{mgm}$. lithium or $23 \mathrm{mgm}$. boron per c.c. of emulsion, and these amounts of isotopes of low natural abundance are costly, the technique is expensive unless the isotope can be readily recovered after plate exposure.

Two series of spectrographic analyses have, therefore, been made on the solutions from the usual processing sequence on nuclear plates coated with $200 \mu$ thickness of emulsion and loaded with natural lithium sulphate and boric acid respectively. It was found that pre-soaking for one hour in distilled water extracted 90 per cent of the boric acid, the remainder being found in the developer ( $50 \mathrm{~min}$. development). In the case of lithium sulphate, two-thirds was found in the pre-soak, one-third in the developer and a small amount in the 10 per cent potassium bromide stop bath (30 min.). There was no detectable boron or lithium in the remaining solutions or in the gelatin, so almost complete recovery can be achieved very simply from the pre-soak and developer solutions.

\section{L. Smrth}

Electromagnetic Separation Group, General Physics Division,

Atomic Energy Research Establishment, Harwell, Berks.

Oct. 1.

\section{Crystal Structure and Magnetic Properties of Cobalt Ferrite at Low Temperatures}

CoBalt ferrite (CoO. $\left.\mathrm{Fe}_{2} \mathrm{O}_{3}\right)$ and magnetite $\left(\mathrm{FeO} . \mathrm{Fe}_{2} \mathrm{O}_{3}\right)$ both crystallize at room tomperature with the inversed-spinel-type of structure ${ }^{1}$. A magnetic transition consisting of an abrupt decrease of saturation magnetization takes place in magnetite ${ }^{2}$ 